\title{
Toxicokinetics and toxicodynamics of paraquat accumulation in mouse brain
}

\author{
Kavita Prasad $^{\mathrm{a}, \mathrm{d}}$, Elizabeth Tarasewicz ${ }^{\mathrm{a}, \mathrm{d}}$, Jason Mathew ${ }^{\mathrm{b}, \mathrm{d}}$, Pamela A. Ohman Strickland ${ }^{\mathrm{c}}$, \\ Brian Buckley $^{\mathrm{d}, \mathrm{e}}$, Jason R. Richardson ${ }^{\mathrm{b}, \mathrm{d}}$, and Eric K. Richfield ${ }^{\mathrm{a}, \mathrm{d},{ }^{*}}$ \\ aDepartment of Pathology and Lab Medicine, Robert Wood Johnson Medical School (RWJMS), \\ University of Medicine and Dentistry New Jersey (UMDNJ), Piscataway, NJ 08854, USA \\ ${ }^{b}$ Department of Environmental and Occupational Medicine, Robert Wood Johnson Medical \\ School (RWJMS), University of Medicine and Dentistry New Jersey (UMDNJ), Piscataway, NJ \\ 08854, USA \\ 'Department of Biostatistics, Robert Wood Johnson Medical School (RWJMS), University of \\ Medicine and Dentistry New Jersey (UMDNJ), Piscataway, NJ 08854, USA \\ dEnvironmental and Occupational Health Sciences Institute (EOHSI), USA \\ eRutgers University, Piscataway, NJ 08854, USA
}

\begin{abstract}
Paraquat (PQ) is a potential human neurotoxicant and is used in models of oxidative stress. We determined the toxicokinetics (TK) and toxicodynamics (TD) of PQ in adult mouse brain following repeated or prolonged PQ exposure. PQ accumulated in different brain regions and reached a plateau after $\sim 18$ i.p. $(10 \mathrm{mg} / \mathrm{kg})$ doses and resulted in modest morbidity and mortality unpredictably associated with dose interval and number. PQ had divergent effects on horizontal locomotor behavior depending on the number of doses. PQ decreased striatal dopamine levels after the 18th to 36th i.p. dose $(10 \mathrm{mg} / \mathrm{kg})$ and reduced the striatal level of tyrosine hydroxylase. Drinking water exposure to PQ $(0.03-0.05 \mathrm{mg} / \mathrm{ml})$ did not result in any mortality and resulted in concentration and time dependent levels in the brain. The brain half-life of PQ varied with mouse strain. PQ accumulates and may saturate a site in mouse brain resulting in complex PQ level and duration-related consequences. These findings should alter our risk assessment of this compound and demonstrate a useful, but complex dynamic model for understanding the consequences of PQ in the brain.
\end{abstract}

\section{Keywords}

Parkinson's disease; Dopamine system; Paraquat; Oxidative stress; Toxicokinetics; Toxicodynamics

() 2008 Published by Elsevier Inc.

*Corresponding author: Department of Pathology and Laboratory Medicine, EOHSI, 170 Frelinghuysen Road, Piscataway, NJ 08854, USA.EKR@EOHSI.Rutgers.edu (E.K. Richfield).

There were no competing financial interests in this work. 


\section{Introduction}

Paraquat $\left(1,1^{\prime}\right.$-dimethyl-4, $4^{\prime}$-bipyridinium dichloride, $\left.\mathrm{PQ}\right)$ is a potent and widely used herbicide, defoliant, and desiccant (California DPR (Dept of Pesticide Regulation), 2005; Yamada, 2003). PQ has been widely studied, periodically assessed, and extensively evaluated for possible risks (Yamada, 2003). In supervised trials, PQ has been detected on many fruits, olives (up to $10 \mathrm{mg} / \mathrm{kg}$ ), olive oil, onions, Brussels sprouts (7.3 mg/kg), tomatoes, field beans $(7.6 \mathrm{mg} / \mathrm{kg})$, other vegetables, chick peas $(3.1 \mathrm{mg} / \mathrm{kg})$, corn, processed corn products, and sorghum coarse grits $(69.7 \mathrm{mg} / \mathrm{kg})$. Residual PQ levels in foodstuffs for animals were often much higher (up to $221 \mathrm{mg} / \mathrm{kg}$ ). However, most supervised testing had levels that were less than detectable. PQ residue in unsupervised settings is unknown and of potential grave concern given the international distribution of food, the millions of farmers using PQ, the potential for misuse, and the unknown total amount being used. PQ is stable on frozen foodstuffs for up to 46 months and resistant to microwave heating extraction (Yamada, 2003; Prasad et al., 2007). Previous risk assessments may not have adequately considered the brain in view of recent data demonstrating adverse brain effects in rodents and the accumulation and prolonged retention of PQ in mouse brain (Dinis-Oliveira et al., 2006; Prasad et al., 2007). An epidemiological association has been found between the use of PQ in agriculture and the incidence of Parkinson's disease (Liou et al., 1997; Hertzman et al., 1990). The toxicity of this compound may be related to the production of oxygen radicals and/or to an energy crisis (Winterbourn, 1981; Schmuck et al., 2002; Castello et al., 2007). The acute toxic effects in the lung, liver and kidney (Bullivant, 1966; Smith and Heath, 1976) have been well recognized in humans and animals, but subtle, chronic, or delayed consequences in human or rodent brain are more difficult to demonstrate. Transport into brain has been repeatedly demonstrated in rodents and may occur via a specific neutral amino acid transporter (Shimizu et al., 2001; McCormack and Di Monte, 2003), where it reaches a concentration ten times lower than in peripheral tissues (Barlow et al., 2003; Prasad et al., 2007). The role of mitochondria as the location for the generation of reactive oxygen species (ROS) due to PQ has become of greater interest (Castello et al., 2007; Cocheme and Murphy, 2008).

In our recent study we demonstrated that PQ persisted in the brain of C57BL/6J mice with a prolonged half-life of approximately 1 month, which contributed to its accumulation with repeated doses at shorter intervals (Prasad et al., 2007). The present study was designed to extend those findings by examining the accumulation of $\mathrm{PQ}$ in different mouse brain regions after repeated exposures (TK) and potential toxicodynamic (TD) consequences on the nigrostriatal system including behavioral and biochemical alterations. Initial testing of drinking water exposure is reported. The half-life of $P Q$ also varied in different inbred mouse strains. These data are critical for the assessment of potential human risks from PQ, and for interpreting animal models using this agent to induce oxidative stress in the brain.

\section{Materials and methods}

\section{Paraquat administration and tissue acquisition}

C57BL/6J (B6J) and other strains of mice were purchased from the Jackson Laboratory

(JAX, Bar Harbor, Maine) for the first and fourth experiments (Experiment I and IV) and 
internally bred using JAX mice for the second and third experiments (Experiment II and III, Table 1). Mice were housed in a climate and light controlled (12/12 h light/dark, light on at 6 A.M.) room at the University of Medicine and Dentistry of New Jersey (UMDNJ). Food and water were provided ad libitum. Animal use procedures were approved by the UMDNJ Institutional Animal Care and Use Committee (IACUC) and were in accordance with the National Institutes of Health Guide for the Care and Use of Laboratory Animals. Care was taken to reduce discomfort or pain in mice. Male mice between the ages of 8-12 weeks were used in Experiments I, II, and IV. Mice up to 40 weeks of age were used in Experiment III. The paraquat dichloride trihydrate (Sigma Chemicals, St. Louis, MO) solution was made fresh in saline for each injection. PQ prepared for drinking water was made fresh 1-2x per week until stability tested and then once per week thereafter.

In Experiment I, 70 mice were randomly divided into two groups of 35 mice each and were treated either $2 \times$ or $3 \times$ per week i.p. Mice were treated at the same time each day with 10 $\mathrm{mg} / \mathrm{kg}$ body weight of PQ or a comparable volume of saline. Mice in the two groups ( $2 \times$ or $3 \times /$ wk) were divided into 5 subgroups which were administered a total of $6(n=5), 12(n=$ $5), 18(n=5)$, or $24(n=10)$ doses of PQ or $24(n=10)$ doses of saline for a total of 35 mice per dosing frequency $(2 \times$ or $3 \times / w k)$. Animal weight was recorded before each injection. Animals receiving 24 doses of PQ $(2 \times$ or $3 \times / w k)$ or saline were tested for their locomotor behavior on selected days of injection and $24 \mathrm{~h}$ later (see below). The mice used for behavioral testing were also tested 1 week after the last treatment prior to sacrifice.

Experiment II was designed to confirm the finding of accumulation of PQ in brain. In experiment II, 55 mice were originally divided into 11 groups of 5 mice each and were given $10 \mathrm{mg} / \mathrm{kg}$ body weight of PQ or saline $3 \times /$ week i.p. For unclear reasons mice in this group started losing weight and many died within the first 7 doses. The surviving mice were reorganized, with the remaining animals now dosed $2 \times$ a week beginning after the 7 th dose. Mice ( $n=4$ or 5/group) were sacrificed 1 week after 7, 12, 18, 24, 30, or 36 doses of PQ or after 18 or 36 doses of saline.

Experiment III tested if PQ could be safely and effectively administered orally in drinking water. Three concentrations of PQ were prepared, low $(\sim 0.03 \mathrm{mg} / \mathrm{ml})$, medium $(\sim 0.04 \mathrm{mg} /$ $\mathrm{ml})$, and high $(\sim 0.05 \mathrm{mg} / \mathrm{ml})$ and administered continuously for either 8 weeks or 12 weeks. Four male mice were included in each of those 6 groups at the beginning of the exposures $(n$ $=24$ total) and group status did not change. Mice were weighed $1-2 \times /$ week during exposures and on the day of sacrifice. Water consumption was also measured 1-2× per week. Mice were sacrificed during the day after consuming PQ daily without a washout period, except that most consumption was during the night.

Experiment IV determined the half-life of PQ in striatum following a single i.p. dose (10 $\mathrm{mg} / \mathrm{kg}$ ) to five different inbred strains ( $n=4$ per strain and time point). We measured the striatal level of PQ after 4, 14, 28, and 56 days.

All mice, except in Experiment IV, were allowed to survive for 1 week after the last i.p. injection. Mice were sacrificed by cervical dislocation. In experiment I, brains were harvested and dissected on ice to obtain the regions of interest (two striata, STR); these were 
immediately frozen with dry ice, whereas the remaining caudal hindbrain with midbrain was fixed in $4 \%$ paraformaldehyde (PFA). Frozen tissues were stored at $-80{ }^{\circ} \mathrm{C}$ until used in assays described below. In experiments II-IV, all regions of interest including frontal cortex (FCtx), striata (STR), hippocampus (Hpc), cerebellum (Cbl) and ventral midbrain (VM) containing substantia nigra (SN) were immediately frozen using crushed dry ice for later use.

\section{Paraquat determination}

The paraquat determination was performed exactly as previously described (Prasad et al., 2007). Briefly, tissue samples (10-60 mg accurately weighed) were placed into a $1.5 \mathrm{ml}$ centrifuge tube and were mixed with $0.15 \mathrm{ml}$ of $12 \%$ acetic acid and then sonicated for approximately $20 \mathrm{~min}$. Samples over $45 \mathrm{mg}$ were not adequately extracted with this volume and were discarded. The centrifuge tubes were placed in microwave vessels (CEM HP500) and heated in the oven (CEM MARS) for $30 \mathrm{~min}$ at $50 \%$ power. The samples were then centrifuged using a $10 \mathrm{kDa}$ filter (Nanosep $10 \mathrm{k}$ Omega Pall Trincor, Exton PA) and the filtrate transferred to HPLC/autosample vials and stored at $-30{ }^{\circ} \mathrm{C}$ until analysis. Paraquat was separated by HPLC (Waters Alliance, Waters Corporation, Waltham, MA) using a ONYX, $4.6 \mathrm{~mm} \times 50 \mathrm{~mm}, 2 \mu \mathrm{m}$ (Phenomenex, Torrance, CA) and quantified using a calibration curve of PQ standards (Sigma Chemicals, St. Louis, MO). Quantification was carried out using LCQ ITMS mass spectrometer (Finnigan, San Jose, CA) equipped with an electrospray ionization source (ESI) and operated using Xcalibur 1.3 software.

Quantification of PQ was based on integrated peak areas of the $186 \mathrm{~m} / \mathrm{z}$ ion in Selective Ion Monitoring (SIM) mode with isolation width $1 \mathrm{~m} / \mathrm{z}$.

In Experiment I, each striatal sample was extracted and quantified independently. In Experiments II-IV each sample was extracted independently, but quantified after combining aliquots from each group member. For most regional measurements (striatum, frontal cortex, cerebellum, and hippocampus), an equal volume aliquot was taken from each of four samples at the same exposure and measured together as a population mean. This pooling for a population mean was done for cost and time savings of analysis since the variance has been well established for our use of this assay. For the hippocampus PQ value at 36 doses in experiment II, individual samples were assessed to provide a confirmatory assessment of variance.

To test the stability of PQ in drinking water, samples were tested from drinking bottles immediately after preparation (fresh) and 3, 4, and 7 days after preparation at the three intended concentrations (low, medium, and high).

\section{Locomotor activity}

Automated locomotor activity chambers equipped with infrared photobeams (Opto-Varimex Minor, Columbus Instruments International Corp., Columbus, $\mathrm{OH}$ ) were used to quantify locomotor activity. Photobeam breaks were recorded each minute for $45 \mathrm{~min}$ for horizontal, vertical, and ambulatory movements. Mice were habituated to the locomotor activity chambers in three 45-min sessions occurring on consecutive days, with all mice receiving i.p. saline injections prior to the session. After the third habituation session, PQ treatment 
began, and the effects on locomotor activity were assessed immediately and $24 \mathrm{~h}$ after injections 6,12, 18 and 24. Activity was also measured 1 week following the last PQ exposure to determine the persistence of effects. Mice were handled by the same individual throughout the course of behavior and each mouse received an injection (saline or PQ) prior to each session during habituation, acute exposure, recovery days, and 7 days after the last exposure.

\section{HPLC-EC determination of catecholamines}

High-performance liquid chromatography (HPLC) analysis of catecholamines by electrochemical detection (HPLC-EC) was performed as described previously (Richardson et al 2006). Briefly, dissected striata were sonicated in $0.1 \mathrm{M}$ perchloric acid (PCA) containing $347 \mu \mathrm{M}$ sodium bisulfite and $134 \mu \mathrm{M}$ EDTA. Homogenates were centrifuged at $15,000 \times g$ for $20 \mathrm{~min}$ at $4{ }^{\circ} \mathrm{C}$, the supernatant removed, and filtered through a $0.22 \mu \mathrm{m}$ filter by centrifugation at $15,000 \times g$ for $20 \mathrm{~min}$. The supernatants were then analyzed for levels of DA, DOPAC, and HVA, as well as for 5-HT and 5-HIAA. Catecholamine concentrations were determined using a Waters Alliance HPLC system equipped with an Antec Leyden electrochemical detector. Quantification was made by reference to calibration curves obtained from individual monoamine standards. Values are reported as $\mathrm{ng} / \mathrm{mg}$ wet weight of tissue.

\section{Western blot analysis}

Western blot analysis was performed as previously described (Richardson et al. 2006). Briefly, striatal samples were homogenized in buffer ( $320 \mathrm{mM}$ sucrose and $5 \mathrm{mM}$ HEPES with $1 \mu \mathrm{g} / \mathrm{ml}$ of the protease inhibitors aprotinin, leupeptin, and pepstatin). Homogenized samples were centrifuged at $2000 \times g$ for $5 \mathrm{~min}$, and the supernatant was centrifuged at $30,000 \times g$ for $30 \mathrm{~min}$. The final pellet was resuspended in homogenization buffer and subjected to polyacrylamide gel electrophoresis on $10 \%$ precast NuPage gels (Invitrogen, Carlsbad, CA). Samples were electrophoretically transferred to a polyvinylidene difluoride membrane, and nonspecific sites were blocked in $7.5 \%$ nonfat dry milk in Tris-buffered saline (135 mM NaCl, $2.5 \mathrm{mM} \mathrm{KCl,} 50 \mathrm{mM}$ Tris, and 0.1\% Tween 20, pH 7.4). Membranes were then incubated first with the appropriate $1^{\circ}$ antibody in Tris-buffered saline with $2 \%$ nonfat dry milk. The $1^{\circ}$ antibody was detected using a goat horseradish peroxidase $2^{\circ}$ antibody followed by enhanced chemiluminescence. The chemiluminescent signal was captured on an Alpha Innotech Fluorchem imaging system and stored as a digital image. Membranes were then stripped for $15 \mathrm{~min}$ at $25^{\circ} \mathrm{C}$ with Pierce Stripping Buffer and reprobed with additional $1^{\circ}$ and $2^{\circ} \mathrm{Abs}$. The $1^{\circ} \mathrm{Ab}$ first detected $\mathrm{TH}$ (rabbit polyclonal, 1:1000, Chemicon, Temecula, CA), followed by the a-tubulin (mouse monoclonal, 1:1000, Sigma-Aldrich, St. Louis, MO). a-Tubulin blots were used to determine protein loading across samples and to normalize changes in protein abundance for all other $1^{\circ} \mathrm{Abs}$. Bands were quantified to O.D., background subtracted, and normalized to their respective value for a-tubulin used as a loading control to yield a ratio. Ratios from two gels containing different samples were combined for analysis. Both gels had samples from all exposures. 


\section{Statistical analysis}

For the analysis of behavioral outcomes, we examined differences between treatment (PQ and saline) over cumulative doses of $\mathrm{PQ}$ using repeated measures analysis of variance with a 3-way factorial design. Behavioral data recorded on the day of dosing and on the recovery day were examined in separate models (see below). The base model included the three-way interaction between frequency ( $2 \times$ or $3 \times /$ week), treatment (PQ or saline), and dose number $(1,6,12,18$ and 24) and all lower order terms. An appropriate correlation structure was chosen by comparing the Akaike Information Criteria (AIC). Backwards stepwise regression, starting with the model including the three-way interaction, between frequency ( $2 \times$ or $3 \times /$ week), treatment (PQ or saline), and dose number $(1,6,12,18$ and 24$)$ and all lower order terms, was then used to select the most appropriate model for this data. Once a final model was obtained, the treatment-by-session interaction was tested to compare the effect of PQ on changes in activity over time relative to saline and post-hoc contrasts were used to compare effects of treatment at each specified dose number. These analyses were performed using SAS/STAT software (SAS system for Windows, Version 9.1.3) (Littell and Milliken, 2006).

Similar analyses were used to examine the weight data, with mouse age (days), treatment, and frequency as predictors. A growth modeling approach was taken, with a random slope for age to allow for inter-mouse variability in growth trajectories. In addition, plots suggested the additional need for a quadratic term for age. Only ages (days) with measures of weight recorded for both frequencies ( $2 \times$ and $3 \times /$ week) were used in this analysis. Thus, data for this analysis included weights measured on each mouse 17 times between 60 and 117 days of age. These analyses were performed using SAS/STAT software (SAS system for Windows, Version 9.1.3 (SAS Institute Inc.)).

Behavioral data analyzed on habituation day 3 and 7 days after the last dose used two-way ANOVA implemented in SigmatStat 3.1 (San Jose, CA).

Levels of catecholamines and TH quantified by Western blots were analyzed using one or two factor ANOVA with treatment frequency and number of doses as between group factors implemented in SigmatStat 3.1 (San Jose, CA). For one measure (levels of HVA in Experiment II), a formal test of normality rejected the null hypothesis that the data was normally distributed. In this case, the Kruskall-Wallis analysis of variance was conducted, with post-hoc Dunn's comparisons. All other post-hoc assessments were carried out based on main effects or interactions as appropriate when the overall effect was significant. For all analyses, initial $P$ values of $₫ 0.05$ were considered statistically significant and post-hoc Holm-Sidak comparisons were performed that controlled for the number of comparisons made.

The kinetics of PQ in different strains was assessed using multiple linear regression with mouse strain as a categorical variable and time as continuous. An interaction between strain and time was tested to evaluate whether the slopes (representing changes over time) were significantly different for the different strains of mice. Residual diagnostics were used to assess the fit of the model. Log transformations were considered as a means to satisfy the assumptions of the model. 


\section{Results}

\section{Effect of i.p. PQ treatment on weight and survival}

Body weight was monitored throughout the duration of experiments I and II. During the implementation of experiment I there were no observed treatment-related changes in group average body weight (Figs. 1A-D). Some mice that died experienced weight loss for one or two doses prior to death. All PQ treated groups gained weight on average. The $3 \times /$ week groups (Figs. 1A-C) received their total number of doses in a shorter time compared to the $2 \times /$ week groups. The $3 \times /$ week group finished their 24 doses in 8 weeks (at $\sim 120$ days of age), whereas the $2 \times$ /week group finished in 12 weeks (at $~ 144$ days of age). There were four mortalities during experiment I (out of 70 total and 50 treated with PQ) with one mouse dying in the $2 \times /$ week PQ group (after dose 15 ) and 3 animals dying in the $3 \times /$ week group (after doses 12,14, and 14). There were no deaths between doses 16 and 24.

Weight data statistically analyzed after completion demonstrated the auto-regressive correlation structure, with correlation depending on the number of days in between measurements, provided the best fit for the data (AIC $=1848.4$ vs. 2066.4 for compound symmetry; unstructured correlation matrix was not estimable). Including the random slope for age also provided a better fit of the model to the data (AIC $=1848.4$ vs. 1889.5 without the random slope). After backwards selection, the final model included the treatment-by-age interaction as well as linear and quadratic terms for age. The overall effect of PQ on changes in weight over time was significantly different than saline (treatment-by-age interaction $F=$ $14.25, \mathrm{~d} f=1.756, p=.0002$ ). Differences in trends due to treatment were apparent at later ages, but not at earlier times, and were present primarily in the PQ 2×/week group (Fig. 1D).

The advantage of the $3 \times /$ week protocol to be completed sooner than the $2 \times /$ week suggested it might be a preferable paradigm. Experiment II was therefore designed to use that dosing frequency. However, we observed significant weight loss and mortality early during treatment which caused us to change to the $2 \times /$ week paradigm to finish the study. A total of 20 deaths occurred before or just after our dosing change at dose 7 with deaths occurring after dose $5(n=2), 6(n=9)$ and $7(n=9)$. After changing to the $2 \times /$ week dosing paradigm after the 7th dose no further animals suffered from weight loss and there were no further mortalities.

There were no observable differences in appearance between groups in their home cage or during locomotor activity.

\section{Brain regional $P Q$ level}

PQ levels were measured in different brain regions from random mice in Experiments I and. II (Fig. 2). In experiment I, each sample was quantified separately whereas in Experiment II, group means were assessed for all regions and doses examined except for the hippocampus after 36 doses (which were analyzed separately). In experiment I, analyses were done using balanced samples $(n=2-4)$ from a striatum of mice treated either $2 \times /$ week or $3 \times /$ week for 6 , 12,18 , or 24 doses. There were no differences in PQ levels due to frequency of dosage at any dose number. In the 24 dose group, the values for the $2 \times /$ week $(1.98 \pm 0.99 \mathrm{ng} / \mathrm{mg}$ tissue, $n=4)$ and $3 \times /$ week $(1.83 \pm 0.92 \mathrm{ng} / \mathrm{mg}$ tissue, $n=4)$ were not significantly different ( $t$-test, $p$ 
$=0.73$ ). In Experiment I (Fig. 2), results were combined for the two dosing frequencies. The conclusion from Experiment I whether additional doses of PQ resulted in continued accumulation or reached a plateau in the striatum was unclear and necessitated experiment II for interpretation. Experiment II demonstrated that the striatal level of PQ reached a plateau after $\sim 18$ doses (Fig. 2). We also used tissue from Experiment II to measure the level of PQ in frontal cortex, hippocampus, and cerebellum after the selected number of doses (18 and 36). PQ levels were: frontal cortex $(n=2)$ after 18 doses $(0.91 \mathrm{ng} / \mathrm{mg})$, hippocampus $(n=4)$ after 18 doses $(0.79 \mathrm{ng} / \mathrm{mg})$ and 36 doses $(0.77 \pm 0.09 \mathrm{ng} / \mathrm{mg})$, and cerebellum $(n=4)$ after 18 doses $(0.63 \mathrm{ng} / \mathrm{mg})$.

\section{Locomotor activity}

Locomotor behavioral analysis was done using a subset of mice from Experiment I ( $n=10$ per group initially). Mice were habituated to the locomotor chambers on each of 3 consecutive days and horizontal, ambulatory, and vertical activities were obtained over a 45 min period. After analysis on the third habituation day, mice were then assigned to four treatment groups ( $\mathrm{PQ}$ or saline given $2 \times$ or $3 \times /$ week) to ensure similar baseline activity levels (Fig. 3A). There were no significant differences between groups on the third habituation day (two-way ANOVA, $F_{1,39}=1.01, p=0.62$ ). Locomotor activity was then measured immediately and $24 \mathrm{~h}$ after the 1st, 6th, 12th, 18th, and 24th injection (dose number) and lastly 7 days after the 24th injection before harvesting tissue. Total horizontal activity counts immediately after (Fig. 3B) and $24 \mathrm{~h}$ after (Fig. 3C) the indicated number of doses demonstrated PQ dose dependent changes locomotor activity only on the day of treatment. There were no significant differences between dosing $2 \times$ or $3 \times /$ week in any behavioral measure.

On treatment the day, the unconstrained correlation structure provided the best fit for the behavioral data (AIC $=3147.4$ vs. 3189.4 for autocorrelation and 3201.1 for compound symmetry). After backwards selection, the final model for behavior included the treatmentby-session and frequency-by-session interactions and all main effects. The overall effect of PQ on changes in activity over time was significantly different than saline (treatment-bysession interaction $F_{4,37}=6.02, p=0.0008$ ). Activity was significantly different between treatments at sessions (total doses) 1, 6, 18 and 24, with activity due to PQ being significantly less in the earlier two sessions (doses 1 and 6) and significantly greater in the last two sessions (doses 18 and 24). There were significant declines after the 1st (70\% of saline) and 6th ( $80 \%$ of saline) dose of PQ compared to saline (post-hoc $p s<0.021$ ). Immediately after the 18 th (144\% of saline) and 24 th (128\% of saline) dose, activity was significantly elevated after PQ compared to saline (post-hoc $p s<0.036$ ).

On the recovery day $24 \mathrm{~h}$ later, the unconstrained correlation structure provided the best fit for the data (AIC $=3359.6$ vs. 3361.8 for autocorrelation and 3370.6 for compound symmetry). After backwards selection, the overall effect of PQ on changes in activity on the recovery day over time was found not to differ significantly from saline (treatment-bysession interaction $F_{4,38}=1.95, p=0.12$ ). 
There was an overall significant difference between groups 7 days after the final dose (Fig. $3 \mathrm{D}$, two-way ANOVA $F_{1,36}=9.03, p=0.005$ ). Horizontal activity was significantly higher in the PQ groups (131\% of saline) compared to the saline groups (post-hoc $p=0.005$ ).

\section{Striatal dopamine and metabolites}

Striatal dopamine (DA) and metabolites from Experiments I and II were measured 7 days after the last PQ treatments (Figs. 4A, B). Following Experiment I approximately the same number of mice treated either $2 \times$ or $3 \times /$ week in each group ( $n=4-8$ per group) found no differences based on dosing frequency and values were combined. The ANOVA from experiment I demonstrated a significant overall effect of PQ only on the level of striatal DA $\left(F_{4,25}=11.77, p<0.001\right)$. Post-hoc pairwise comparisons (Holm-Sidak) revealed marginally significant higher striatal level of DA 1 week after the 12th $(p=0.025,118 \%$ of saline) and 18th ( $p=0.044,116 \%$ of saline) PQ treatment compared to saline and significantly lower DA 1 week after the 24th treatment compared saline ( $p<0.001,76 \%$ of saline, Fig. 4A). No other significant PQ-related changes occurred with HVA, DOPAC, 5HT, 5-HIAA, NE, or DA turnover, although the level of HVA after 24 doses was reduced to a similar magnitude as that of DA ( $75 \%$ of saline).

Following Experiment II, the ANOVA also demonstrated a significant overall effect of PQ on the level of striatal DA $\left(F_{6,34}=14.09, p<0.001\right)$. Post-hoc pairwise comparisons (Holm-Sidak) demonstrated significantly lower levels of DA after PQ treatments 18, 24, 30, and 36 compared to saline (all $p s<0.0001,71-80 \%$ of saline, Fig. 4B). ANOVA also demonstrated a significant overall effect of PQ on the level of striatal DOPAC $\left(F_{6,34}=6.32\right.$, $p<0.001$ ). Post-hoc pairwise comparisons (Holm-Sidak) demonstrated significant decreases in DOPAC after the 30th and 36th doses (both $p<0.0001$ ). The Kruskall-Wallis analysis of variance was conducted, and demonstrated a significant overall effect of PQ on the level of striatal HVA (H\# 27.56 with $6 \mathrm{~d} f, p<0.001$ ). Post-hoc pairwise comparisons (Dunn's method) demonstrated significant decreases in DOPAC after the 24th, 30th and 36th doses (all $p s<0.05$ ). No significant PQ-related changes occurred with 5-HT or 5-HIAA.

\section{Western analysis}

Striatal TH was measured in mice from Experiment I 7 days after 0 (saline controls), 6, 12, 18, or 24 PQ treatments (Fig. 5) using striata not used for PQ measurements or HPLC.

Approximately the same number of mice treated either $2 \times$ or $3 \times /$ week treatments were used in each group ( $n=5-10$ per each PQ dose group) and no difference was observed based on dosing frequency and values were combined. Samples from mice receiving a different number of doses were distributed equally over two gels. There was an overall effect of PQ on the striatal level of the tyrosine hydroxylase (TH) protein (ANOVA, $F_{4,38}=4.20, p=$ 0.007). Post-hoc pairwise comparisons (Holm-Sidak) revealed significantly lower striatal level of TH after the 6th $(p<0.001)$ and 12th $(p=0.007)$ PQ treatments compared to saline. The largest decrease in TH occurred after 6 doses with a linear recovery after subsequent doses. 


\section{$P Q$ exposure in drinking water}

PQ was stable in drinking water for at least 1 week. The mean concentrations of the freshly made PQ were $0.30 \pm 0.002 \mathrm{mg} / \mathrm{ml}$ (low), $0.42 \pm 0.002 \mathrm{mg} / \mathrm{ml}$ (medium), and $0.47 \pm 0.003$ $\mathrm{mg} / \mathrm{ml}$ (high). No decline in the concentration of PQ was seen after 3, 4, or 7 days in drinking water. Combining PQ concentrations from 3, 4, and 7 days in drinking water using the three targeted concentrations yielded values similar to fresh PQ, $0.30 \pm 0.002 \mathrm{mg} / \mathrm{ml}$ (low), $0.41 \pm 0.002 \mathrm{mg} / \mathrm{ml}$ (medium), and $0.47 \pm 0.003 \mathrm{mg} / \mathrm{ml}$ (high). Change in weight over time was not significantly different between the three groups of mice during PQ exposure (Two Way Repeated Measures ANOVA, One Factor Repetition, $F_{2,359}=0.172, P=0.843$ ). There was a significant effect of aging on weight $\left(F_{16,359}=67.347, P<0.001\right)$. There was a suggestion of weight loss and/or slowed weight gain in the groups between 2 and 5 weeks of PQ exposure (Fig. 6). There were no fatalities. PQ was measured in the striatum from each of the six groups and suggested that brain levels were related to the concentration of $\mathrm{PQ}$ in drinking water and duration of exposure (Fig. 7) TD outcomes were not planned for this first long term oral exposure.

\section{Brain half-life of $P Q$ in different strains}

Appropriateness of residual diagnostics from the linear regression did not differ whether one used the raw value of PQ (ng/mg of tissue) or the log-transformed value. However, the logtransformed value of PQ improved the apparent linearity with time. The interaction between strain and time was significant ( $p=0.0094, F_{4,10}=6.10$ ), indicating that the slopes differed significantly between strains (Fig. 8). Strain NOD/LtJ differed significantly from all other strains (comparing to $129 \mathrm{~S} 1 / \mathrm{EiJ}, p=0.0071$; to $\mathrm{A} / \mathrm{J} p=0.0020$; to C57BL/6J, $p=0.0018$; and to $\mathrm{PWK} / \mathrm{PhJ}, p=0.0416$ ). No other pair of strains differed significantly from one another. Elimination from brain was linear for each strain (C57BL/6J $r^{2}=0.91,129 \mathrm{~S} 1 / \mathrm{EiJ} r^{2}$ $\left.=0.99, \mathrm{~A} / \mathrm{J} r^{2}=0.95, \mathrm{NOD} / \mathrm{LtJ} r^{2}=0.83, \mathrm{PWK} / \mathrm{PhJ} r^{2}=0.90\right)$. The elimination half life in brain was shortest in strain C57BL/6J ( 1 month) and longest in NOD/LtJ ( $\sim 3$ months).

\section{Discussion}

\section{Toxicokinetics}

Data from this study support and extend our findings regarding the accumulation and persistence of PQ in mouse brain (Prasad et al., 2007). The charged PQ was shown here to accumulate to similar levels in different regions of brain (striatum, frontal cortex, hippocampus, and cerebellum) after systemic exposure. A similar plateau level of PQ was found after two independent experiments using a similar number of doses $(\sim 18)$. This was an unexpected and important finding and will be needed for interpreting the adverse consequences of PQ in mouse brain in this paper (Fig. 9) and work by others. The cellular and subcellular location(s) for the accumulation and reason for the long half-life of PQ in brain are not yet known, but their identification will help in determining potential mechanisms of action. It is already known from our in vivo work and the work of others in vivo and in vitro that neurons are adversely affected (Prasad et al., 2007; Richardson et al., 2005; Dinis-Oliveira et al., 2006; Schmuck et al., 2002; Yang and Tiffany-Castiglioni, 2005, 2007). This may be due to direct, indirect, or complex effects on neurons. A role for the adverse effect of PQ on microglia has been reported (Wu et al., 2005). The subcellular 
location for PQ in cells is also not known at this time, but recent in vitro data implicate mitochondria as one important organelle for both the location of PQ and the consequences of ROS generation (Castello et al., 2007; Cocheme and Murphy, 2008). A variety of unidentified membrane transporters will be required for the transport of $\mathrm{PQ}$ from the circulation across the blood brain barrier (BBB), the plasma membrane, and the mitochondrial membrane. Possible transporters across the BBB have been suggested (Shimizu et al., 2001; McCormack and Di Monte, 2003; Barlow et al., 2003) and diet may play a role in PQ uptake into the circulation and brain. Cellular and mitochondrial transporters have been suggested, but not clearly established (Cocheme and Murphy, 2008; Barlow et al., 2003). A role for the dopamine transporter has been frequently mentioned because of the close structural similarity between PQ and MPP+, but ruled out by experimental data (Barlow et al., 2003; Richardson et al., 2005). The reason for the difference between brain and other organs in transporting or accumulating PQ in mice have not been identified, but may contribute to organ vulnerability. In addition to the unique TK aspects of $\mathrm{PQ}$ in adult brain, we have also shown that fetal and neonatal brain are exposed to PQ following gestational or lactating dam exposure (Barlow et al., 2007).

We have previously shown that PQ had a linear pattern of elimination with a half-life in brain of approximately 1 month in adult C57BL/6J mice after a single dose $(10 \mathrm{mg} / \mathrm{kg})$ resulting in a low brain level (Prasad et al., 2007). The half-life following a low brain level (following a single $10 \mathrm{mg} / \mathrm{kg}$ dose) varied from one to 3 months among different inbred strains (C57BL/6J, NOD/LtJ, 129S1/EiJ, A/J, PWK/PhJ) with the C57BL/6J strain having the shortest half-life of mice. The half-life may be longer or nonlinear when starting at a higher brain level as a consequence of repeated exposure. The accumulation and long-half life will contribute to the complex TD in brain. A low brain level has been shown to produce adverse consequences including an elevated lipid peroxidation found to persist for up to 1 month (Prasad et al., 2007). Five doses of PQ resulted in an intermediate brain level and a reduction in the functional activity of the proteasome 1 week after the last dose (Prasad et al., 2007). Since PQ reaches a plateau after $\sim 18$ i.p. doses, consequences after 18 doses will reflect the additional duration of exposure as the level may not increase.

Perhaps of potential greater significance were the identification of brain accumulation following oral exposure in drinking water and the variable half-life due to genetic background. A low concentration of PQ in drinking water $(\sim 0.3 \mathrm{mg} / \mathrm{ml})$ resulted in a daily consumption in mice of $\sim 1.2 \mathrm{mg}$ ( $\sim 4 \mathrm{ml} /$ day of water) or a total of $\sim 67.2 \mathrm{mg}$ resulting in a brain concentration of $\sim 0.12 \mathrm{ng} / \mathrm{mg}$ after 8 weeks of exposure. This can be contrasted with brain levels following single dose gavage of $10(0.25 \mathrm{mg}), 20(0.50 \mathrm{mg})$ and $50(1.25 \mathrm{mg})$ $\mathrm{mg} / \mathrm{kg}$ resulting in brain levels of $0.07 \pm 0.01,0.12 \pm 0.05$ and $0.27 \pm 0.02 \mathrm{ng} / \mathrm{mg}$, respectively. The reason for the higher brain levels following gavage compared to drinking water is unclear. They may relate to differences in PQ absorption due to the presence of food in the GI tract as most rodents eat and drink at similar times during the night (Kissileff, 1969). Higher concentrations or longer exposures in drinking water resulted in higher brain levels. The level in brain was not demonstrated to reach a plateau and the levels were lower than those seen following systemic exposure. Brain levels of PQ similar to those seen with systemic exposure might be expected with higher PQ concentrations in water and/or longer 
exposures. No mortality was seen with this route and concentrations. In addition, we showed that PQ was stable in drinking water for up to 1 week. We have previously demonstrated PQ is stable to microwave extraction and others have shown stability with freezing. Thus, PQ has the potential to be highly stable under certain circumstances. It cannot yet be stated what the lowest level of brain PQ might be adverse in mice nor whether humans are vulnerable to these levels. In addition, the variable half-life due to genetic background (1-3 months) suggests that humans may also have variable brain levels and/or half-lives that might participate in vulnerability or resistance to the effects of PQ. Based on this data, caution may be in order for risk assessment in humans.

\section{Toxicodynamics}

The other major finding of this work was that the striatal concentrations of DA were significantly reduced after 24 doses in both experiments to about the same magnitude ( 70 $80 \%$ of control). Significant changes in DOPAC and HVA were also present in Experiment II after the additional time associated with 24-36 doses. Early changes in striatal catecholamines were predominantly due to DA, which increased after 12 and 18 doses only in Experiment I. Others have failed to identify significant changes in striatal DA despite TH + neuronal loss (McCormack et al., 2002). However, these paradigms might not have exposed mice to sufficient PQ to reach a plateau brain level or not allowed a lower concentration of PQ to act in brain for a long enough time. Thus the level of PQ and time may be critical features for striatal DA loss in addition to the previously identified TH+ neuronal loss occurring at lower doses or after shorter intervals of exposure. We were not able to determine the relationship between striatal DA and nigral $\mathrm{TH}+$ neuron number in these experiments, but this should be a key determination for the future.

The mortality resulting from PQ has been previously reported and reflects consequences on lung and/or other organs (Smith, 1985; Keeling et al., 1981; Smith et al., 1978). In Experiment I, doses were given $2 \times$ or $3 \times$ /week and resulted in the same timing of mortality (between doses 12 and 15) that suggests mortality was not due solely to accumulation of PQ or duration of exposure because of the absence of mortality after 15 doses. In Experiment II, unexpected early mortality was seen using the $3 \times$ /week injection paradigm (after 5, 6, or 7 doses). The reason(s) for this dramatic difference in mortality in Experiment II associated with both an increased frequency and the occurrence after fewer doses compared to Experiment I was unclear. The same age and gender of mice were used in both experiments. The same person handled and injected mice using the same source of PQ. This source of PQ was also used for calibrating our mass spectrometer during measurements and determining extraction efficiencies and we did not detect degradation or errors in dosing. The same strain of mouse was used (C57BL/6J). The only difference we identified was that Experiment I mice were purchased directly from a supplier and in Experiment II mice were bred internally from breeders purchased from the same supplier. This difference may relate to the composition of rodent chow used in the two facilities. The increased mortality may also relate to season, gestational effects, or other environmental interaction such as unrecognized stress. However, the differences in mortality should not be used to ignore the similarities in PQ accumulation with dose or the striatal loss of DA after 24 doses in both experiments. These are the two main findings that should be gleaned from this study. Unexplained 
mortality occurs with the systemic administration of other neurotoxicants including MPTP, methamphetamine, and rotenone, mortality can vary substantially in magnitude at the same dose, and varies between and within the same lab (Battaglia et al., 2003; Lapointe et al., 2004; Crutchfield and Dluzin, 2006; He et al., 2004). Changing a paradigm during an exposure has been reported and may be justified depending on purpose (Lapointe et al., 2004). We believe that twice weekly i.p. exposures may be a reasonable frequency if intermittent systemic exposures are desired, but may still result in mortality or morbidity. A better approach, in terms of human exposure, may be an oral drinking water exposure to generate any desired brain PQ concentration up to a brain plateau level. Neither weight loss nor mortality were a feature of our initial drinking water exposure. Consideration should be given to the relevance and purpose of the method of PQ exposure in mice.

The effect of PQ on locomotor activity has similarities and differences from those reported previously. The reduction following a first dose of $\mathrm{PQ}$ has been demonstrated and may relate to acute effects on DA release (Thiruchelvam et al., 2000a,b). This reduction in activity was also seen after the 6th dose. There was a steady increase in horizontal activity measured at the time of dosing after the first dose such that mice eventually became hyperactive after the 18th and 24th doses which persisted 7 days after the last dose of PQ. These changes in horizontal activity following more than 12 doses have not been previously reported. The acute changes in locomotor activity after systemic PQ may relate in part to the measured changes in striatal dopamine or to changes not yet measured related to the release or reuptake of DA or effects of DA on striatal dopamine receptors. The locomotor response pattern over time resembles a sensitization-like behavior (the increase in function (locomotor activity) due to a drug or chemical (PQ) when it is used repeatedly) that might be mediated in part by striatal DA or DA receptors. In vivo voltammetry or the measurement of striatal dopamine receptors might clarify the mechanism for delayed hyperactivity. The use of alternative behavioral measures and longer durations of PQ exposure could also be considered.

The pattern of TH protein loss in the striatum differed from the pattern of DA changes during Experiment I. The decline in the level of TH was greatest after 6 doses and gradually increased with subsequent doses of PQ, although the level of TH did return to that of the saline exposed mice. The pattern of DA differed being elevated slightly at early doses and then declining at later doses. The dissociation between TH and DA levels in the striatum and the dynamic changes in locomotor behavior demonstrate the complex biological consequences occurring in the striatum as a consequence of the accumulation and persistence of PQ over time. Disparity between different presynaptic DA markers alone or in relationship to behavioral measures have been observed by others (Georgievska et al., 2002; Haycock et al., 2003; Kogan et al., 1976; Simon et al., 1994; Nishi et al., 1989; Bowyer et al., 1992). Compensatory changes in nigrostriatal system have been noted by others. These dynamic and changing outcomes over time support the need to measure multiple outcomes over time and the importance of relating TD responses to TK data. 


\section{Summary}

The TK and TD of PQ in mouse brain are complex. The TK reflect the accumulation and plateau level of PQ in brain and are related to the long half-life. The TD consequences are brain PQ level and duration dependent (Fig. 9). Future descriptions of brain consequences from $P Q$ need to be related to brain level and duration of PQ since little data is currently available with this critical information. The adverse level or half- life of PQ in humans cannot be speculated at this time, but further research will be critical to determine safe levels of exposure for this commonly used herbicide.

\section{Acknowledgments}

We acknowledge support for this work from the National Institute of Environmental Health Sciences (NIEHS) for R21 ES01183, P30 ES005022-19, and R01ES-15991.

\section{Abbreviations}

$\begin{array}{ll}\text { ANOVA } & \text { analysis of variance } \\ \text { BBB } & \text { blood brain barrier } \\ \text { FCtx } & \text { frontal cortex } \\ \text { PD } & \text { Parkinson's disease } \\ \text { PDP } & \text { Parkinson's disease phenotype } \\ \text { PQ } & \text { paraquat } \\ \text { ROS } & \text { reactive oxygen species } \\ \text { SN } & \text { substantia nigra } \\ \text { STR } & \text { striatum } \\ \text { TK } & \text { toxicokinetics } \\ \text { TD } & \text { toxicodynamics } \\ \text { VM } & \text { ventral midbrain }\end{array}$

\section{References}

Barlow BK, Bennice L, Thiruchelvam MJ, Cory-Slechta DA, Ballatori N, Richfield EK. Selective dithiocarbamates increase synaptosomal dopamine content, brain concentration of paraquat and correlate with potentiation of MPTP and paraquat toxicity. J Neurochem. 2003; 85:1075-1086. [PubMed: 12716439]

Barlow, BK.; Kochar, J.; Prasad, K.; Buckley, B.; Mirochnitchenko, O.; Richfield, EK.; Thiruchelvam, M. Paraquat exposure during periods of neurodevelopment and the Parkinson's disease phenotype. Soc Neurosci Abstract Poster \# 25417/M10; San Diego, CA. Session \# 254, Parkinson's Disease Models and Mechanisms II; 2007.

Battaglia G, Busceti CL, Pontarelli F, Biagioni F, Fornai F, Paparelli A, Brunio V, Ruggieri S, Nicolett F. Protective role of group-II metabotropic glutamate receptors against nigro-striatal degeneration induced by 1-methyl-4-phenyl-1,2,3,6-tetrahydropyridine in mice. Neuropharmacology. 2003; 45:155-166. [PubMed: 12842121]

Bowyer FJ, Tank AW, Newport GD, Slokker W Jr, Ali SF, Holson RR. The influence of environmental temperature on the transient effects of methamphetamine on dopamine levels and 
dopamine release in rat striatum. J Pharmacol Exp Ther. 1992; 260(2):817-824. [PubMed: 1346646]

Bullivant CM. Accidental poisoning by paraquat: report of two cases in man. Br Med J. 1966; 1:12721273. [PubMed: 5939816]

California DPR (Dept of Pesticide Regulation). Summary of Pesticide Use Report Data 2005 Indexed by Commodity. 2005. p. 1-495.

Castello PR, Drechesl DA, Patel M. Mitochondria are a major source of paraquat-induced reactive oxygen species production in the brain. J Biol Chem. 2007; 282(19):14186-14193. [PubMed: 17389593]

Cocheme HM, Murphy MP. Complex I is the major site of mitochondrial superoxide production by paraquat. J Biol Chem. 2008; 283(4):1786-1798. [PubMed: 18039652]

Crutchfield KC, Dluzin DE. Rotenone produces opposite effects upon mouse striatal dopamine function as a result of environmental temperature. Neurotox Res. 2006; 91:15-21. [PubMed: 16464748]

Dinis-Oliveira RJ, Remiao F, Carmo H, Duarte JA, Sanchez Nararro A, Bastos ML, Carvalho F. Paraquat exposure as an etiological factor of Parkinson's disease. Neurotoxicology. 2006; 26:1110-1122. [PubMed: 16815551]

Georgievska B, Kirik D, Björklund A. Aberrant sprouting and down-regulation of tyrosine hydroxylase in lesioned nigrostriatal dopamine neurons induced by long-lasting overexpression of glial cell line derived neurotrophic factor in the striatum by lentiviral gene transfer. Exp Neurol. 2002; 177:461-474. [PubMed: 12429192]

Haycock JW, Becker L, Ang L, Furukawa Y, Hornykeiwicz O, Kish SJ. Marked disparity between age-related changes in dopamine and other presynaptic dopaminergic markers in human striatum. $\mathrm{J}$ Neurochem. 2003; 87:574-585. [PubMed: 14535941]

He J, Xu H, Yang Y, Zhang X, Li X-M. Neuroprotective effects of olanzapine on methamphetamineinduced neurotoxicity are associated with an inhibition of hyperthermia and prevention of Bcl-2 decrease in rats. Brain Res. 2004; 1018(2):186-192. [PubMed: 15276877]

Hertzman C, Wiens M, Bowering D, Snow B, Calne D. Parkinson's disease: a case-control study of occupational and environmental risk factors. Am J Ind Med. 1990; 17:349-355. [PubMed: 2305814]

Keeling PL, Pratt IS, Aldridge WN, Smith LL. The enhancement of paraquat toxicity in rats by $85 \%$ oxygen: lethality and cell-specific lung damage. Br J Exp Pathol. 1981; 62(6):643-654. [PubMed: 7326219]

Kissileff HR. Food-associated drinking in the rat. J Comp Physiol Psychol. 1969; 67:284-300. [PubMed: 5787379]

Kogan FJ, Nichols WK, Gibb JW. Influence of methamphetamine on nigral and striatal tyrosine hydroxylase activity and on striatal dopamine levels. Eur J Pharmacol. 1976; 36(2):363-371. [PubMed: 6286]

Lapointe N, St-Hilaire M, Martinoli M-G, Blanchet J, Gould P, Rouillard C, Cicchetti F. Rotenone induces non-specific central nervous system and systemic toxicity. FASEB J. 2004; 18:717-719. [PubMed: 14766796]

Liou HH, Tsai MC, Chen CJ, Jeng JS, Chang YC, Chen SY, Chen RC. Environmental risk factors and Parkinson's disease: a case-control study in Taiwan. Neurology. 1997; 48:1583-1588. [PubMed: 9191770]

Littell, RC.; Milliken, GA. SAS System for Mixed Models. SAS Institute, Inc; Cary, NC: 2006.

McCormack AL, Di Monte DA. Effects of L-dopa and other amino acids against paraquat-induced nigrostriatal degeneration. J Neurochem. 2003; 85:82-86. [PubMed: 12641729]

McCormack AL, Thiruchelvam M, Manning-Bog AB, Thiffault C, Langston JW, Cory-Slechta DA, Di Monte DA. Environmental risk factors and Parkinson's disease: selective degeneration of nigral dopaminergic neurons caused by the herbicide paraquat. Neurobiol Dis. 2002; 10(2):119-127. [PubMed: 12127150]

Nishi K, Kondo T, Narabayashi H. Difference in recovery patterns of striatal dopamine content, tyrosine hydroxylase activity, and total bipterin content after 1-methyl-4-phenyl-1,2,3,6- 
tetrahyroperidine administration: a comparison of young and older mice. Brain Res. 1989; 489(1): 157-162. [PubMed: 2568151]

Prasad K, Winnik B, Thiruchelvam MJ, Buckley B, Mirochnitchenko O, Richfield EK. Prolonged toxicokinetics and toxicodynamics of paraquat in mouse brain. Environ Health Perspect. 2007; 15(10):1448-1453. [PubMed: 17938734]

Richardson JR, Quan Y, Serer TB, Greenamyre JT, Miller GW. Paraquat neurotoxicity is distinct from that of MPTP and rotenone. Toxicol Sci. 2005; 88(1):193-201. [PubMed: 16141438]

Richardson JR, Caudle WM, Wang M, Dean ED, Pennell KD, Miller GW. Developmental exposure to the pesticide dieldrin alters the dopamine system and increases neurotoxicity in an animal model of Parkinson's disease. FASEB J. 2006; 20:1695-1697. [PubMed: 16809432]

SAS Institute Inc. User's Guide. Cary, NC: SAS/STAT® 9.1.

Schmuck G, Rohrdanz E, Tran-Thi QH, Kahl R, Schluter G. Oxidative stress in rat cortical neurons and astrocytes induced by paraquat in-vivo. Neurotoxicol Res. 2002; 4(1):1-13.

Shimizu K, Ohtaki K, Matsubara K, Aoyama K, Vezono T, Saito O, Suno M, Ogawa K, Hayase N. Carrier-mediated processes in blood-brain barrier penetration and neural uptake of paraquat. Brain Res. 2001; 906(1):135-142. [PubMed: 11430870]

Simon JR, Richter JA, Ghetti B. Age-dependent alterations in dopamine content, tyrosine hydroxylase activity, and dopamine uptake in the striatum of the Weaver mutant mouse. J Neurochem. 1994; 62(2):543-548. [PubMed: 7905025]

Smith LL. Paraquat toxicity. Philos Trans R Soc London. 1985; 311:647-657. [PubMed: 2869523]

Smith P, Heath D. Paraquat: Crit. Rev Toxicol. 1976; 4(4):411-445.

Smith LL, Rose MS, Wyatt I. The pathology and biochemistry of paraquat. CIBA Found Symp. 1978; 65:321-341. [PubMed: 38952]

Thiruchelvam M, Brockel BJ, Richfield EK, Baggs RB, Cory-Slechta DA. Potential and preferential effects of combined paraquat and maneb on nigrostriatal dopamine systems: environmental risk factors for Parkinson's disease. Brain Res. 2000a; 873:225-234. [PubMed: 10930548]

Thiruchelvam M, Richfield EK, Baggs RB, Tank AW, Cory-Slechta DA. The nigrostriatal dopaminergic system as a preferential target of repeated exposures to combined paraquat and maneb: implications for Parkinson's disease. J Neurosci. 2000b; 20(24):9207-9214. [PubMed: 11124998]

Winterbourn CC. Production of hydroxyl radicals from paraquat radicals and $\mathrm{H}_{2} \mathrm{O}_{2}$. FEBS Lett. 1981; 128(2):339-342. [PubMed: 6894903]

Wu X, Block M, Zhang W, Qin L, Wilson B, Zhang W, Veronesi B, Hong JS. The role of microglia in paraquat-induced dopaminergic neurotoxicity. Antioxid Redox Signal. 2005; 7:654-661. [PubMed: 15890010]

Yamada, Y. Paraquat (057): First Draft. 2003. p. 533-698.http://www.fao.org/ag/AGP/AGPP/Pesticid/ JMPR/Download/2004_eva/paraquat.pdf

Yang W, Tiffany-Castiglioni E. The bipyridl herbicide paraquat produced oxidative stress-mediated toxicity in human neuroblastoma SH-SY5Y cells: relevance to the dopaminergic pathogenesis. J Toxicol Environ Health. 2005; 68:1939-1961.

Yang W, Tiffany-Castiglioni E. The bipyridl herbicide paraquat induces proteasome dysfunction in human neuroblastoma SH-SY5Y cells. J Toxicol Environ Health. 2007; 70:1849-1857. 

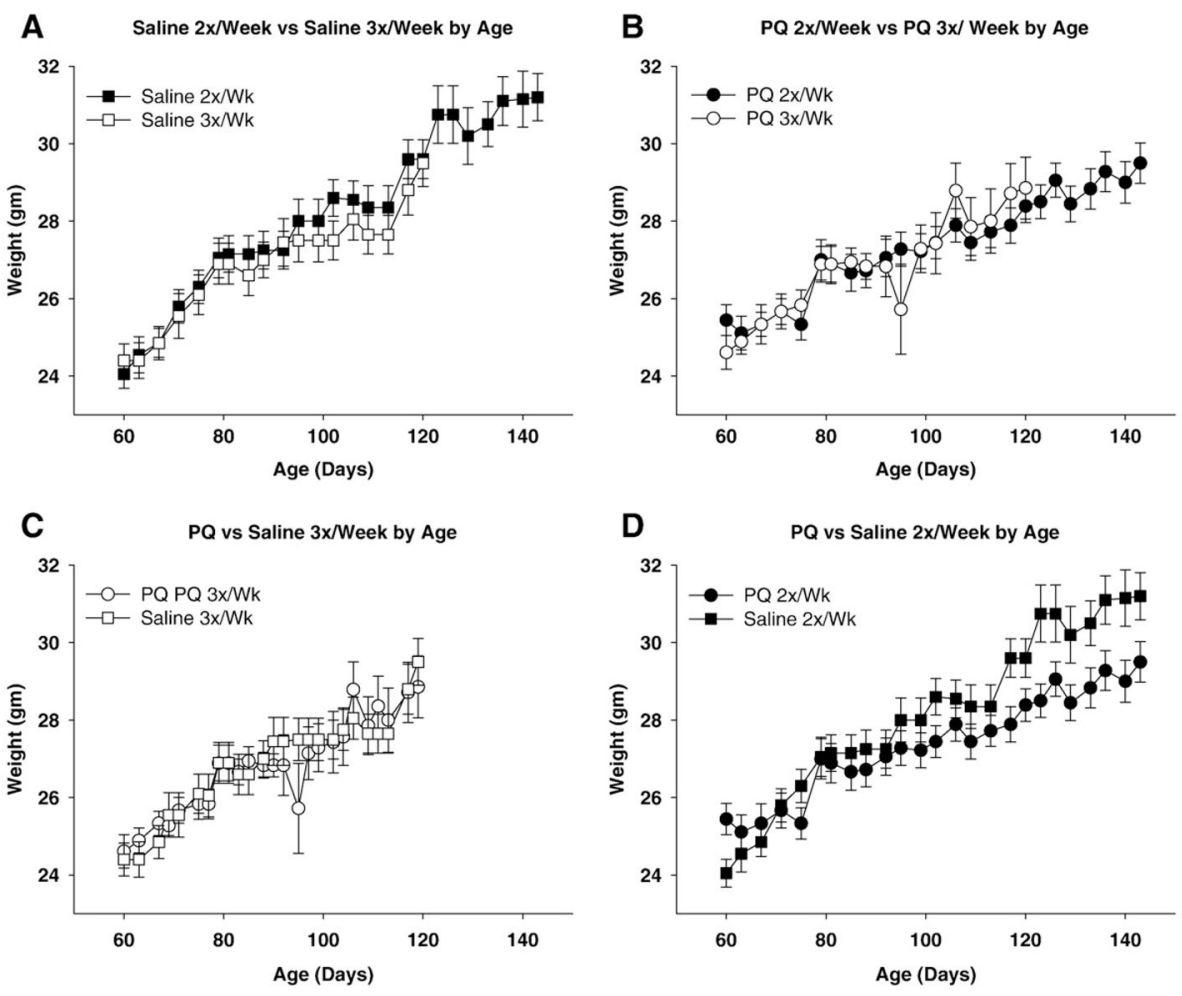

Fig. 1.

Changes in body weight for the two saline and two PQ groups treated either $2 \times$ or $3 \times /$ week for 24 total doses in experiment I ( $n=10$ /group initially). There was no difference in weight gain comparing the two saline groups (A) or the two PQ groups (B) up to the time of sacrifice for the $3 \times /$ wk group ( $\sim 120$ days). There was a statistical difference in PQ versus saline treatment which was observed only at a later age when dosed $2 \times /$ week (D), but not observed with 3x/week (C). Symbols: - - PQ 2x/week, $\bigcirc-P Q 3 \times /$ week, $\mathbf{\square}$ - saline $2 \times /$ week, $\square$ - saline $3 \times /$ week. 


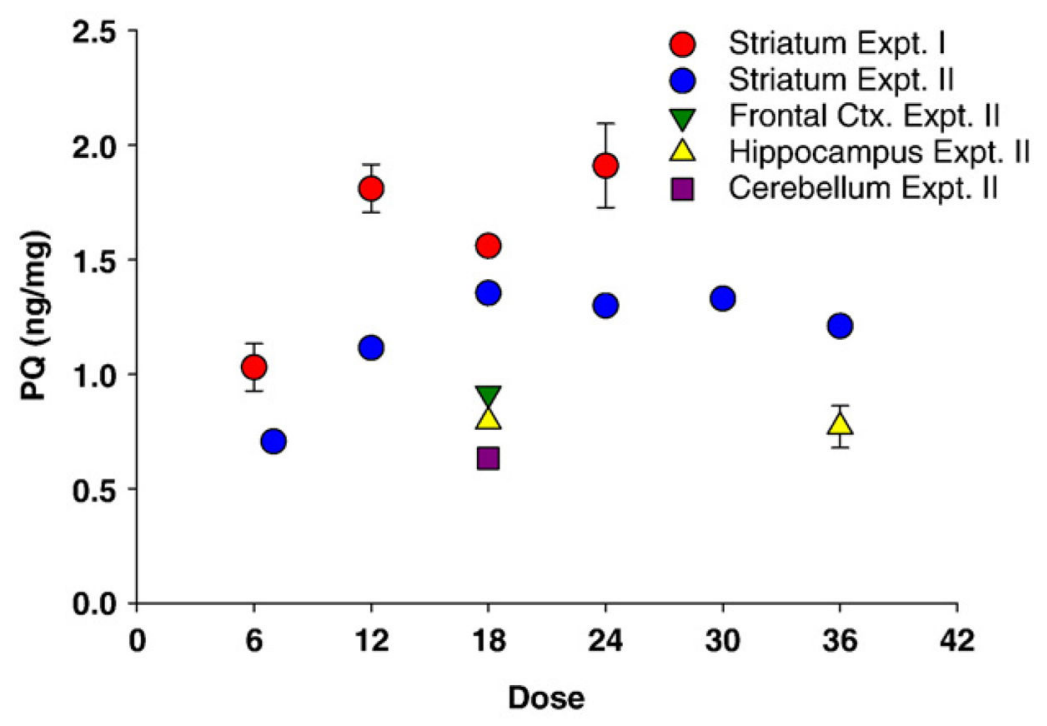

Fig. 2.

Level of PQ in brain regions. In Experiment I ( $\bullet$ ) PQ was measured in striatal samples after $6(n=4), 12(n=4), 18(n=4)$, or $24(n=8)(10 \mathrm{mg} / \mathrm{kg})$ doses. There were no differences between the levels of PQ measured after dosing 2 xor $3 \times /$ week and were combined. In Experiment II, the PQ level in striatum $(n=4, \bullet)$ was measured after a total of 7, 12, 18, 24, 30 , and $36(10 \mathrm{mg} / \mathrm{kg})$ doses. PQ was also measured after the indicated number of doses from frontal cortex $(n=2, \boldsymbol{\nabla})$, hippocampus $(n=4, \Delta)$, and cerebellum $(n=4, \mathbf{v})$. Symbols without an error bar are the population mean and error bars indicate the s.e.m. Samples from the striatum in Experiments I and II, frontal cortex, cerebellum, and hippocampus were extracted and analyzed at different times. 

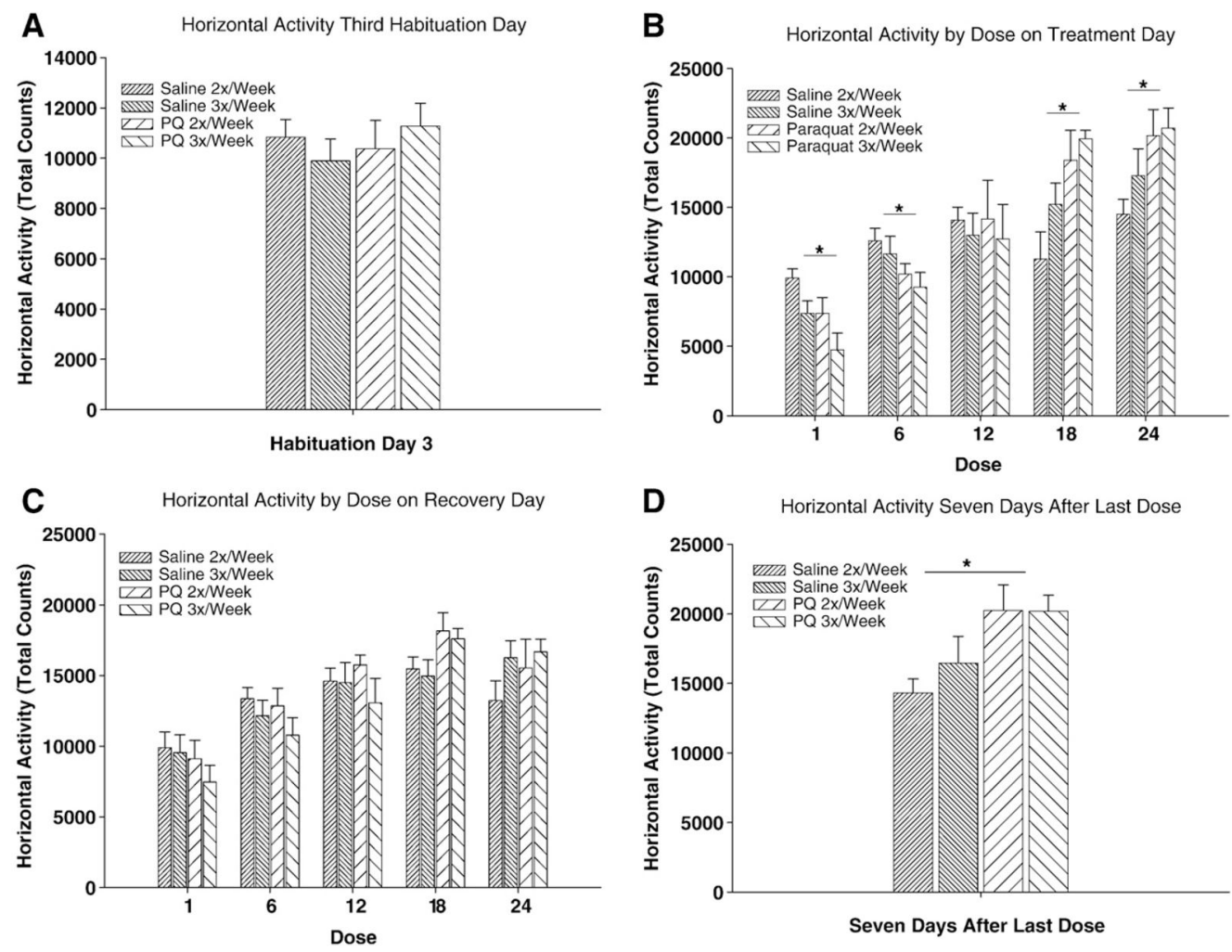

Fig. 3.

Total horizontal activity counts. Mice were allocated to different groups after the third habituation (A) and then treated with either PQ or saline $2 \times$ or $3 \times /$ week ( $n=9-10$ /group) (B-D). No difference was seen in behavior related to treatment frequency so the groups were combined for statistical analysis, but were plotted separately. Activity differed between groups over the course of treatments immediately after the indicated doses (B), but not $24 \mathrm{~h}$ later (C). Mice treated with PQ were also significantly more active 7 days after the final treatment and prior to sacrifice (D). * indicate significant differences between PQ and saline following post-hoc testing. 

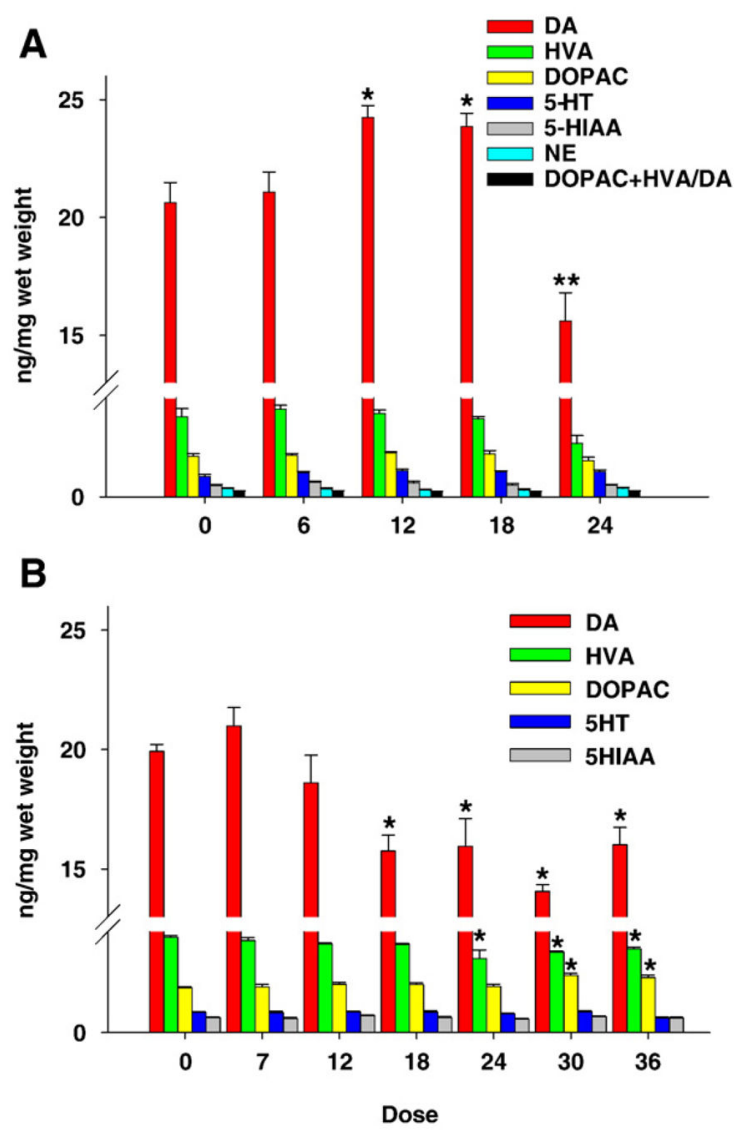

Fig. 4.

Striatal levels of dopamine (DA), homovanillic acid (HVA), 3, 4-dihydroxyphenylacetic acid (DOPAC), serotonin (5-HT), 5-hydroxyindoleacetic acid (5-HIAA) were determined using HPLC in striatal samples 7 days after the indicated doses of PQ. A. The $2 \times(n=4)$ and $3 \times(n=4)$ /week treatments were combined as no significant differences were observed between these groups. Post-hoc testing revealed either a modestly significant increase in DA 7 days after the 12 th and 18 th doses of PQ compared to saline $(* *)$ or a significant decrease in DA after the 24th dose compared to all other groups (*). B. Post-hoc testing demonstrated significant declines in DA after 18 or more doses of PQ, significant declines in HVA after 24 or more doses of PQ, and significant increases in DOPAC after 30 or more doses of PQ compared to saline $(*)$. 


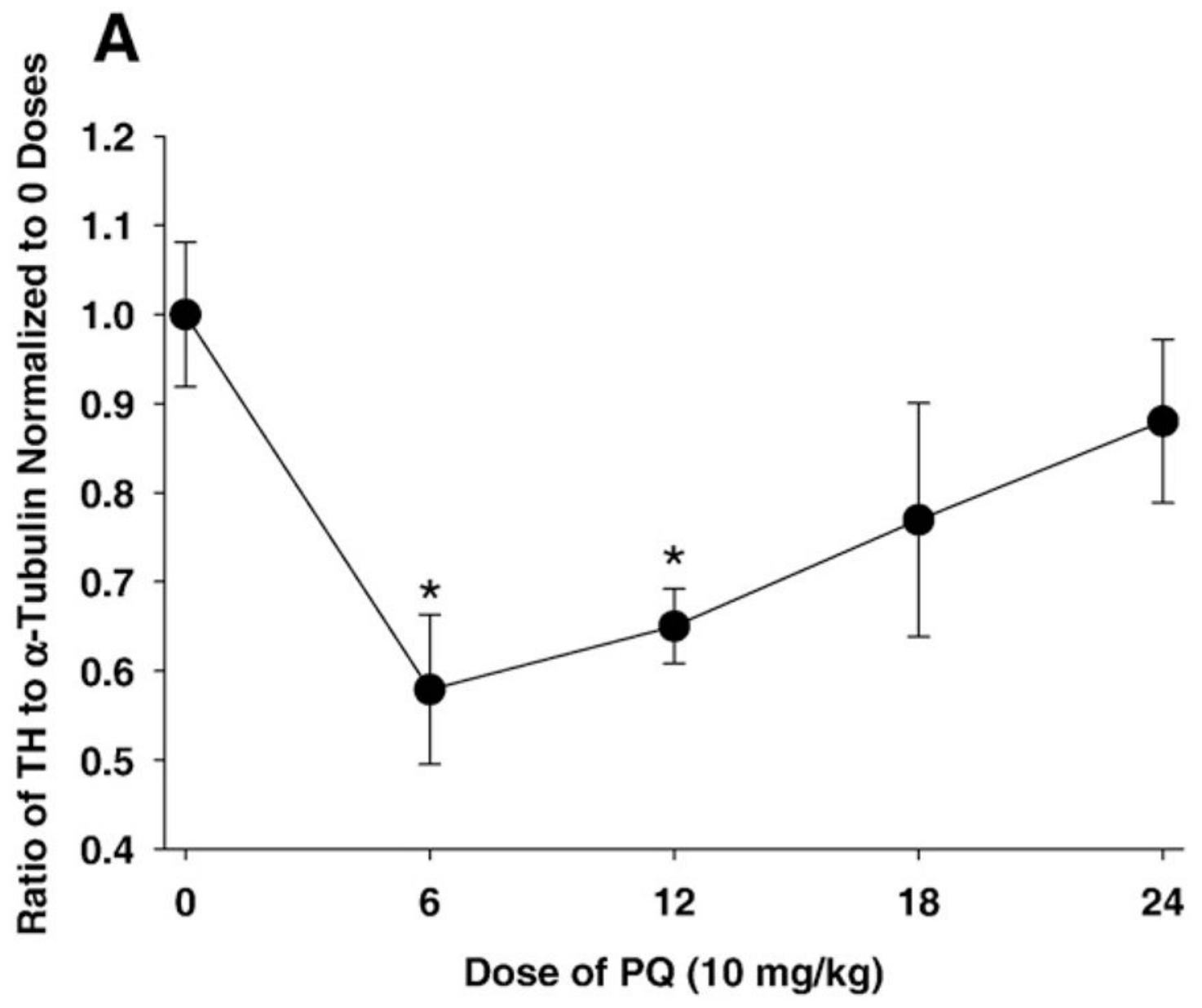

B

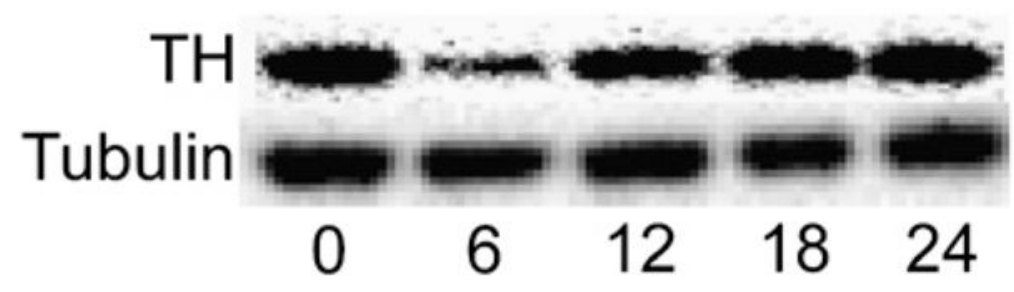

Fig. 5.

Changes in the abundance of striatal TH after the indicated doses. Samples sizes $(n)$ for the different doses of PQ were 0 (10), 6 (8), 12 (7), 18 (5), and 24 (8) as limited by tissue availability. Samples included a similar number of $2 \times$ and $3 \times /$ week doses of PQ and saline. (A) Plot of the ratio of densitometric values for TH to a-tubulin at the indicated number of doses. Values indicated by * were significantly different following appropriate post-hoc tests compared to 0 doses of PQ (saline control group). (B) Representative image of TH and a-tubulin at indicated number of doses of PQ. 


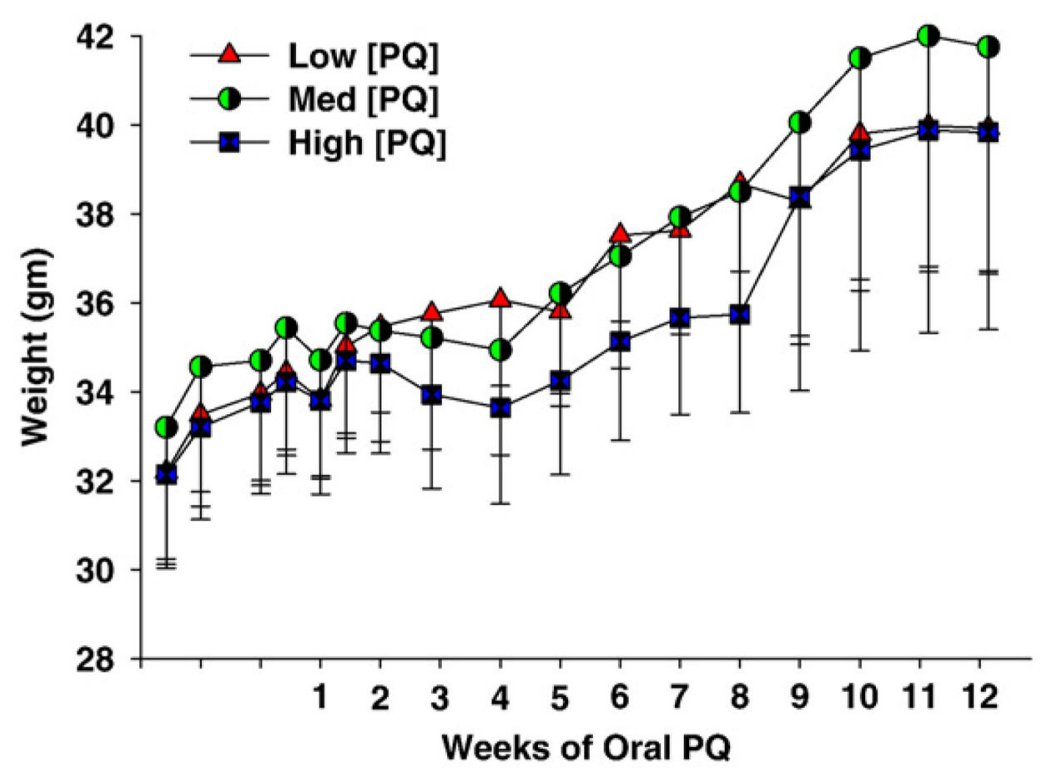

Fig. 6.

Mice $(n=24)$ were randomly assigned to one of six groups ( $n=4$ per group). There were 3 drinking water exposure concentrations: low $(\sim 0.03 \mathrm{mg} / \mathrm{ml})$, medium $(\sim 0.04 \mathrm{mg} / \mathrm{ml})$, and high $(\sim 0.05 \mathrm{mg} / \mathrm{ml})$ for either 8 or 12 weeks. Weights were measured prior to and during PQ exposure. Errors bar are smaller for the first 8 weeks of PQ exposure because of a larger sample size. Mice gained weight following all exposures. 


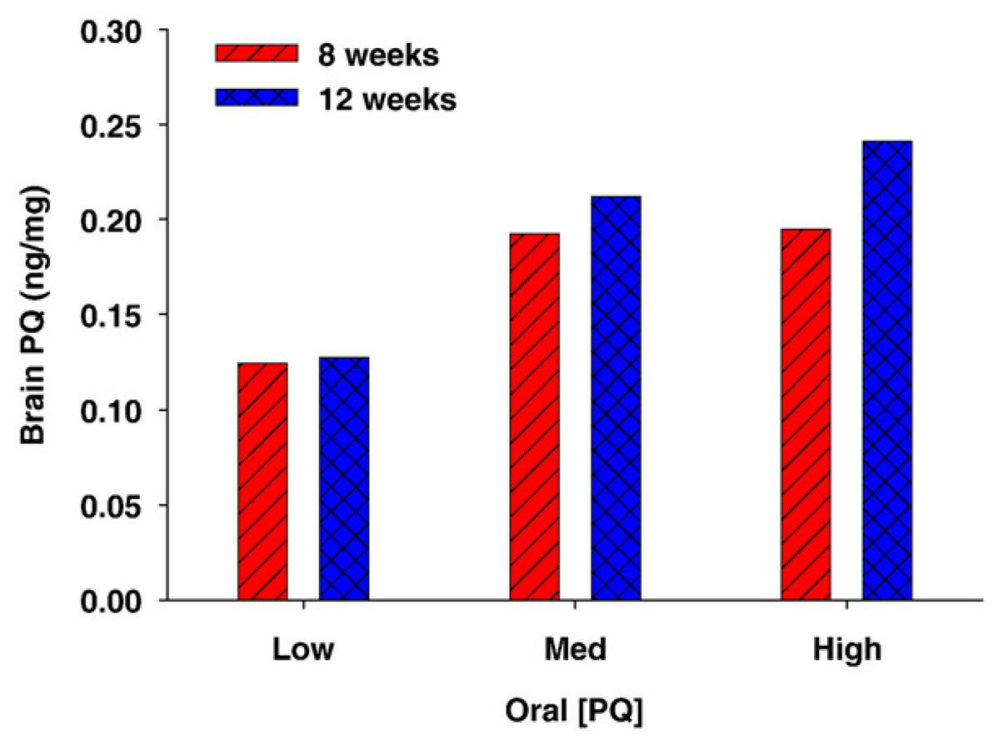

Fig. 7.

The level of PQ was measured in the striatum following low $(0.03 \mathrm{mg} / \mathrm{ml})$, medium $(0.04$ $\mathrm{mg} / \mathrm{ml})$, or high $(0.05 \mathrm{mg} / \mathrm{ml})$ concentration of PQ in drinking water for 8 or 12 weeks. The sample size was 4 for each condition. The level of PQ in the brain was concentration and time dependent. 


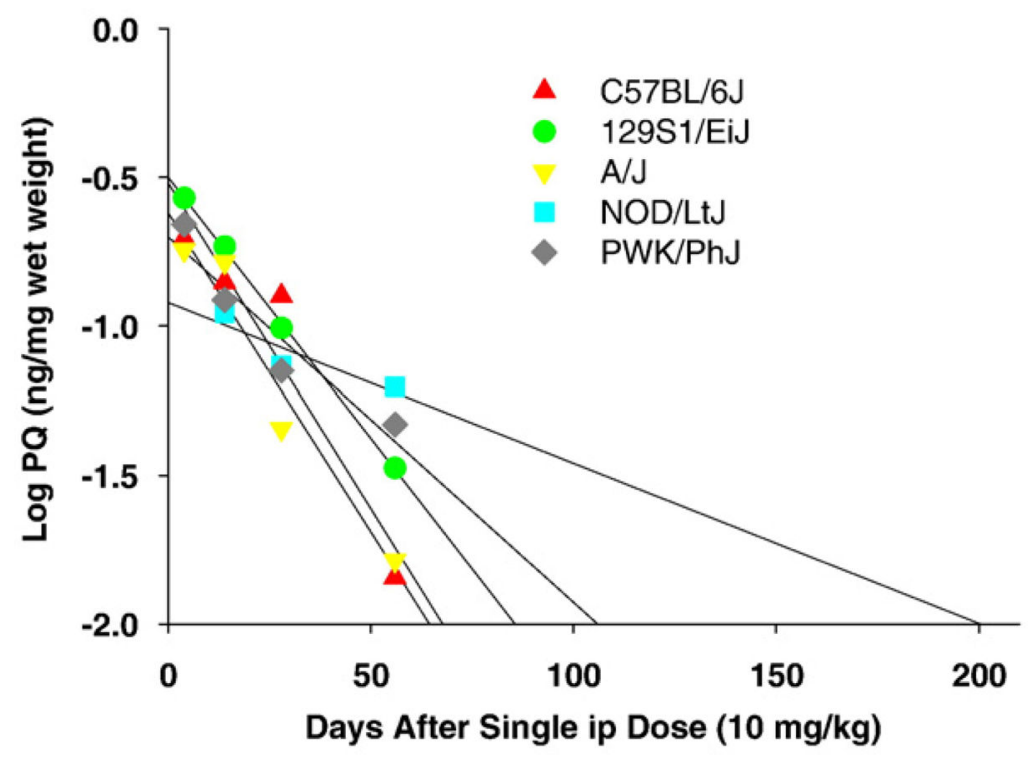

Fig. 8.

Time course of PQ in ventral midbrain (VM) of five different mouse strains. PQ (10 mg/kg) was injected by the intraperitoneal route and tissue harvested at different time points. At no time point was PQ entirely eliminated from brain. (Each value represents a mean from 4 mice.) Elimination followed a linear pattern over time with correlation coefficients ranging from 0.83 to 0.99 for the different strains. 


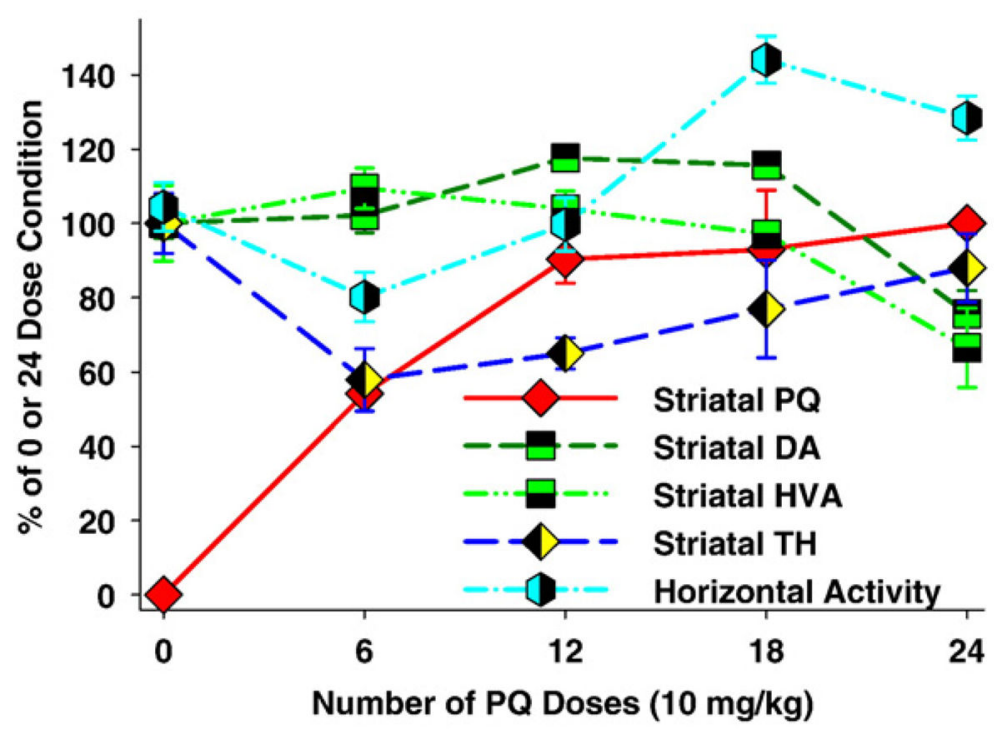

Fig. 9.

Composite graph of effects of PQ by dose and time. Selected data from Experiment I presented in previous figures were normalized to the zero dose (saline) values (DA, HVA, $\mathrm{TH}$ ), the habituation day 3 value (horizontal activity), or the 24th dose (PQ) to allow comparison of effects over time or dose number. PQ accumulated in a linear manner until reaching a level that may have saturated an unknown site(s) in the striatum of mouse brain between the 12th to 18th dose. The brain level of PQ was then maintained with continued exposure. DA and HVA were unaffected or minimally increased between 6 and 18 doses, but then decreased in association with the 24 th dose (1 week later). TH was significantly decreased after the 6th and 12th dose and then slowly and linearly increased to near a baseline level. Horizontal activity was significantly decreased on the day of dosing after the 1st (not shown) and 6th dose of PQ and gradually increased with subsequent doses leading to significantly increased activity after the 18th and 24th doses and 1 week after the last dose (not shown). 


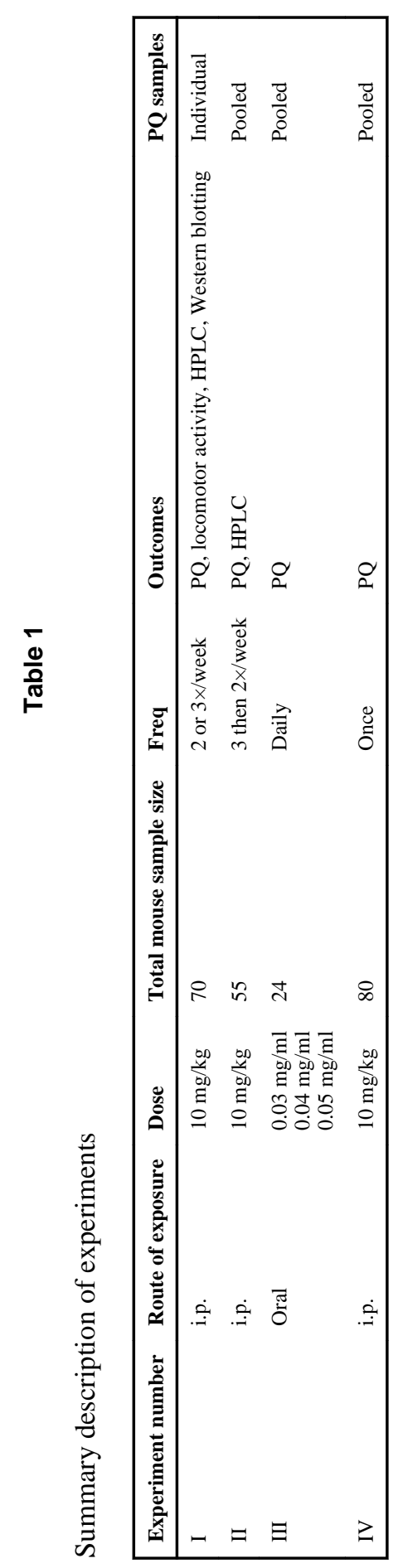

Exp Neurol. Author manuscript; available in PMC 2014 May 11. 